\title{
Scritture della migrazione, postcolonialismo e alterità. Una breve storia della critica
}

Daniele Comberiati

\section{(2) OpenEdition}

1 Journals

\section{Edizione digitale}

URL: https://journals.openedition.org/cher/10729

DOI: 10.4000/cher.10729

ISSN: 2803-5992

\section{Editore}

Presses universitaires de Strasbourg

\section{Edizione cartacea}

Data di pubblicazione: 30 juin 2013

Paginazione: 13-22

ISBN: 978-2-35410-054-4

ISSN: 1968-035X

\section{Notizia bibliografica digitale}

Daniele Comberiati, «Scritture della migrazione, postcolonialismo e alterità. Una breve storia della critica», reCHERches [Online], 10 | 2013, online dal 08 février 2022, consultato il 10 février 2022. URL: http://journals.openedition.org/cher/10729 ; DOl: https://doi.org/10.4000/cher.10729

\section{(c) (i) (2)(2)}

$\mathrm{Ce}(\mathrm{tte})$ œuvre est mise à disposition selon les termes de la Licence Creative Commons Attribution Pas d'Utilisation Commerciale - Partage dans les Mêmes Conditions 4.0 International. 


\title{
Scritture della migrazione, postcolonialismo e alterità. Una breve storia della critica
}

\author{
Daniele Comberiati \\ Université de Bruxelles
}

u Repubblica del primo giugno 2012, in una recensione al romanzo
Timira ${ }^{1}$, scritto a quattro mani da Wu Ming 2 e Antar Mohamed, Massimo Vincenzi parla delle «colonie» come di una «parola quasi rimossa dal nostro vocabolario»². Al di là di alcune critiche a cui l'articolo si presta - nel sottotitolo si parla di Timira come del «nuovo romanzo di Wu Ming» e nel testo Antar Mohamed non è mai interpellato, tanto che a tratti sembra di ritrovarsi nella dinamica fra «autore» straniero e "curatore» italiano tipica della prima fase della letteratura della migrazione -, risulta interessante notare come, con questo romanzo edito da una grande casa editrice (e recensito su un noto quotidiano), più che con il precedente einaudiano L'ottava vibrazione ${ }^{3}$ di Lucarelli, la riproposizione della storia coloniale e la conseguente riflessione postcoloniale abbiano finalmente trovato posto all'interno della letteratura contemporanea tout court, uscendo dal ghetto migrante in cui erano confinate. Negli ultimi anni è possibile notare una sorta di «appropriazione» del tema da parte di diversi scrittori italiani più o meno noti: a parte il caso del già citato Lucarelli, colpisce che nel 2011, proprio in concomitanza con l'adesione italiana all'attacco della Nato in Libia, corollario di una sanguinosa guerra civile (anch'essa interpretabile in un'ottica postcoloniale) e alla caduta del regime di Gheddafi, siano usciti due romanzi che riprendevano - attraverso una chiave interpretativa legata

1 Wu Ming 2 e Antar Mohamed, Timira, Torino, Einaudi, 2012.

2 M. Vincenzi, «Partigiani, esuli e ribelli. La nostra storia di una donna», La Repubblica, 1 giugno 2012, p. 45.

3 C. Lucarelli, L'ottava vibrazione, Torino, Einaudi, 2008. 
all'attualità come aveva già fatto nel 2004 con Ghibli ${ }^{4}$ Luciana Capretti - la cacciata degli italiani dalla Libia, il cui viaggio viene messo in relazione con le odierne peregrinazioni dei migranti attraverso la lingua di Mediterraneo che separa l'Italia dall'antica «quarta sponda» (Mazzantini 20115; Abate 20116). Il romanzo di Margaret Mazzantini, in particolare, pone immediatamente un'autrice di successo (attraverso un'operazione simile a quella effettuata a suo tempo proprio da Lucarelli) con un tema postcoloniale e molto attuale. $\mathrm{Al}$ di là del giudizio di valore su questi testi e delle critiche che si possono muovere all'operazione, è interessante notare come il tema coloniale, con la specifica declinazione libica, inizi ad apparire nella letteratura italiana. Si tratta di pochi esempi, che probabilmente toccano l'opinione pubblica solo in superficie, ma è possibile che tali narrazioni abbiano portato ad un allargamento della discussione coloniale e postcoloniale all'interno del contesto nazionale, e questo anche in ambito letterario. Certo il caso di Timira, almeno nelle intenzioni, è diverso: in tal caso la colonia non è una semplice tematica o un'ambientazione, ma la ragione stessa della presenza dell'autore: il sottotitolo «romanzo meticcio» è volto a sottolineare, oltre alla pluralità delle voci, l'ibridazione attuatasi all'interno dell'identità italiana, oggi modificatasi anche in virtù (o a causa) della sua storia coloniale.

Vincenzi nella sua recensione parlava, non a caso, di «rimosso» coloniale: impiegando un termine tratto dal gergo psicanalitico, egli si mostra vicino a tutta una serie di studi storici che, proprio a partire dall'oblio della storia coloniale (le cui responsabilità sembrano non avere appartenenza politica), hanno cercato di ricostruire le vicende dell'Italia e dei suoi possedimenti d'oltremare ${ }^{7}$. Il concetto di «rimozione» - ovvero qualcosa che è presente, costitutivo dell'io, ma che si vuole celare, anche se di fatto risulta impossibile cancellarlo - è a ben guardare la chiave che ha ispirato il recente documentario di Luca Guadagnino, Inconscio italiano ${ }^{8}$, una delle opere recenti più interessanti sulla nostra esperienza coloniale, che propone il parallelismo fra passato e presente (o, per meglio dire, fra vecchio e nuovo colonialismo), proprio a partire dalla rimozione della violenza attuata.

4 L. Capretti, Ghibli, Milano, Rizzoli, 2004.

5 M. Mazzantini, Mare al mattino, Torino, Einaudi, 2011.

6 A. Abate, Ultima estate in suol d'amore, Vicenza, Neri Pozza, 2011.

7 A. Del Boca, Gli italiani in Africa orientale, voll. I-IV, Milano, Mondadori, 1976-1987; M. Dominioni, Lo sfascio dell'impero, Roma-Bari, Laterza, 2008.

8 Il documentario di Luca Guadagnino, Inconscio italiano, presentato al Festival di Torino nel 2011, si avvale della collaborazione di Angelo Del Boca, Lucia Ceci, Iain Chambers, Michela Fusaschi, Alberto Burgio e Ida Dominijanni, oltre che delle immagini d'archivio dell'Istituto Luce. 
Tale operazione di rimozione - la quale, è bene ribadirlo, non rappresenta un'espulsione, bensì uno spostamento o, per dirla in termini «temporali», un'attesa nell'affrontare una specifica questione - porta a dinamiche interessanti, soprattutto in letteratura: come ha giustamente notato Derobertis ${ }^{9}$, l'avventura coloniale all'interno della discussione culturale italiana non è stata completamente dimenticata, piuttosto appare vittima di un particolare processo di apparizione / sparizione che ne attesta la presenza costante nel tempo, quasi si trattasse di un'ossessione messa da parte ma pronta a tornare in qualsiasi momento.

I cambiamenti recenti che hanno radicalmente modificato la società italiana dagli anni Ottanta a questa parte, hanno certo reso urgente una discussione letteraria che prenda in considerazione, fra le altre cose, la portata postcoloniale del contesto attuale. Vi è infatti una «pratica postcoloniale» riscontrabile in una serie di testi e di modalità di interazione che può essere associata alla letteratura migrante: le storie di viaggio e i tentativi di integrazione degli scrittori appartenenti alla "prima fase», così come la loro interazione/subordinazione con un giornalista straniero, piuttosto che le ultime opere di Lakhous ${ }^{10}$ o di Cristina Ali Farah ${ }^{11}$, appartengono di diritto alla riflessione sul concetto di postcoloniale, soprattutto se a tale termine diamo il senso attribuitogli prima da Bhabha e successivamente, in ambito italiano, ribadito da Mezzadra ${ }^{12}$.

Vi è, accanto alla "pratica» postcoloniale, una serie di spunti teorici (che insieme compongono una variegata e alle volte contraddittoria «teoria») che sono entrati a far parte della riflessione critica in ambito italiano. Analizzando dal punto di vista cronologico l'impiego (o il rifiuto) di alcune nozioni della teoria postcoloniale, è possibile ricostruire, in parte, anche la specifica situazione degli studi accademici e scientifici italiani riguardanti la letteratura della migrazione, che si mostra di fatto il campo privilegiato in cui si è cercato di mettere in pratica tali fondamenti critici.

Prendiamo come punto di partenza la «nascita» critica della letteratura della migrazione in Italia e le prime discussioni al riguardo. Notiamo fin

9 R. Derobertis, Fuori centro. Percorsi postcoloniali all'interno della letteratura italiana, Roma, Aracne, 2010.

10 Cfr. A. Lakhous, Scontro di civiltà per un ascensore a Piazza Vittorio, Roma, E/O, 2006; Divorzio all'islamica a viale Marconi, Roma, E/O, 2010; Un pirata piccolo piccolo, Roma, E/O, 2011.

11 C. Ubax Ali Farah, Madre piccola, Milano, Frassinelli, 2007.

12 S. Mezzadra, La condizione postcoloniale, Verona, Ombre corte, 2008. 
dall'inizio almeno tre differenti approcci, portati avanti tra l'altro da luoghi e discipline diverse, che possono fungere da orientamento per le evoluzioni che vedremo in seguito. Se in genere si fa risalire, dal punto di vista dei testi, la letteratura migrante alla fine degli anni Ottanta (1989) e all'inizio dei Novanta ${ }^{13}$, le prime riflessioni critiche e teoriche sono di qualche anno posteriori.

Il primo approccio, che per diversi anni ha avuto la funzione di critica "canonica», è attribuibile a Armando Gnisci, comparatista allora all'Università La Sapienza di Roma. Il suo testo La letteratura italiana della migrazione ${ }^{14}$ svela immediatamente i referenti teorici: l'ottica «militante» e il fatto di vedere nell'arrivo degli scrittori migranti una sorta di rinnovamento «messianico» della letteratura italiana contemporanea sono elementi evidenti che si ritroveranno, con alcuni aggiustamenti, anche nelle opere successive. Non è un caso che di letteratura «italiana» della migrazione se ne occupi un comparatista: il contesto teorico è quello degli scrittori e intellettuali caraibici e in parte sudamericani (soprattutto Glissant); il rapporto fra gli scrittori nati in Italia e quelli giunti dopo le ondate migratorie degli anni Ottanta è analizzato da un lato attraverso la critica legittima al provincialismo italiano e all'incapacità di «accogliere» l'alterità, e dall'altro attraverso la fiducia nel meticciato e nell'ibridazione, mettendo da parte la conflittualità che emerge nell'incontro fra culture diverse.

La prima italianista a occuparsi della questione è invece Graziella Parati, che inaugura, al di là del discorso teorico, un "posizionamento» geografico indicativo della diaspora intellettuale, in particolare nell'ambito accademico, dell'Italia negli ultimi due decenni. In seguito si potrà notare come saranno diversi gli studiosi italiani che, dall'estero, si interessano alla letteratura migrante e postcoloniale: le ragioni possono essere diverse, e alcune appaiono piuttosto ovvie. Da una parte l'italianista all'estero ha un rapporto diverso con il canone e con le necessità di inserire autori «ufficiali» all'interno del proprio programma. Egli si muove in uno spazio in cui l'italiano è lingua straniera, dunque in una condizione di minorità rispetto ad altre realtà linguistiche, operando una mediazione in primo luogo fra l'italiano e la lingua del paese di accoglienza, in secondo luogo con le altre lingue straniere; è da tale posizione marginale che, per certi versi, risulta più facile intraprendere un percorso relativamente innovativo, perché lo

13 D. Comberiati, Scrivere nella lingua dell'altro. La letteratura degli immigrati in Italia (1989-2007), Bruxelles, Peter Lang, 2010.

14 A. Gnisci, La letteratura italiana della migrazione, Roma, Lilith, 1997. 
scontro con la letteratura «ufficiale» è meno netto. Inoltre va anche detto che spesso questi studiosi agiscono in luoghi in cui le riflessioni sulla migrazione e sul postcolonialismo sono piuttosto avanzate (in particolare negli Stati Uniti, in Inghilterra, in Francia e recentemente in Germania), dunque il contesto teorico del postcolonialismo italiano si inserisce in un discorso più ampio che, per alcuni passi, è già stato percorso. Il caso di Graziella Parati è illuminante: all'interno delle sue opere, a partire da When the 'other' is black ${ }^{15}$, attraversando Margins at the centre ${ }^{16}$ è indagata la relazione della marginalità con il centro, attraverso un percorso teorico che, da Bhabha, giunge fino a Spivak attraversando Said, ovvero toccando i cardini dei maggiori teorici del postcolonialismo. Anche uno degli ultimi saggi, The art of Talking Back in a Destination Culture ${ }^{17}$, riprende il percorso della letteratura legandolo con quello della lingua e del contesto in cui tale produzione è recepita, attraverso un'analisi che interessa anche le teorie della ricezione.

La terza «linea» cui si accennava poc'anzi è quella costituita dalle prime riflessioni di Alessandro Portelli, autore di un saggio molto interessante sul tema ${ }^{18}$. Anglista di formazione ed esperto di storia orale, con i suoi studi ha avuto il merito di mettere in relazione l'esperienza migrante e postcoloniale italiana con tutta una serie di produzioni inglesi e soprattutto americane, spesso cronologicamente antecedenti. Il concetto di «letteratura afroitaliana» da lui coniato sulla falsa riga della letteratura «afro-americana» introduce anche in ambito italiano la questione del "colore» (che diversi critici hanno in seguito ripreso) e soprattutto nelle sue parole riecheggia l'Atlantico nero con cui Paul Gilroy definisce la cultura transnazionale che trova origine nella diaspora nera e nella tratta degli schiavi. Tale visione della letteratura migrante come isola all'interno di un arcipelago molto più vasto mostra anche l'indubbio vantaggio di inserirla in un arco cronologico di lunga durata e di evitare di ridurla a caso eccezionale dovuto all'imponente ondata migratoria recente. La lungimiranza del suo intervento è testimoniata, tra le altre cose, da un convegno tenutosi pochi mesi fa a Lagos, in

15 Cfr. G. Parati, «When the «Other» is Black: Portraits of Africans by Contemporary Italian Writers» in Romance Language Annual 5, West Lafayette, Purdue Research Foundation, 1993, p. 272-277.

16 Cfr. Ead., «Margins at the Centre: African Italian Voices», in Italian Studies in Southern Africa, n. 2, 1995, p. 1-15.

17 Cfr. Ead., Migration Italy: The Art of Talking Back in a Destination Culture, TorontoBuffalo, London, University of Toronto Press, 2005.

18 A. Portelli, Mediterranean Passage: The Beginnings of an African Italian Literature and the African American Exemple in Mapping African America, New York, Oxford University Press, 2000. 
Nigeria, il primo convegno sulla letteratura migrante italiana nell'Africa subsahariana. Dal 2 al 5 aprile 2012, nell'ambito del Lagos Black Heritage Festival Symposium, si è infatti tenuta una conferenza sulle narrazioni afroitaliane del Mediterraneo nero (The Black in the Mediterranean). La scelta di focalizzare il primo evento della serie sulle connessioni tra l'Italia e la Nigeria è singolare perché la Nigeria non è mai stata una colonia italiana (a differenza dei paesi del Corno d'Africa), eppure - come sostengono gli organizzatori dell'evento - la presenza italiana è fortemente sentita in Nigeria e il legame tra i due paesi è evidente se si guarda ai flussi migratori. È un altro esempio di quella "postcolonialità estesa» che contraddistingue la letteratura migrante contemporanea.

La validità e l'attualità degli approcci di Parati e Portelli sono manifestate anche dagli spunti e dalle spore che hanno prodotto. Un volume di Caterina Romeo, al tempo anch'essa negli Stati Uniti, analizzava la letteratura italoamericana attraverso la specola degli studi di genere. Narrative tra due sponde ${ }^{19}$ mette in relazione scrittrici italo-americane di tre generazioni, ma propone un paradigma critico utile anche per studiare la letteratura italiana della migrazione. Memoir di italiane d'America, recita giustamente il sottotitolo, espandendo la definizione di «italiane d'America» attraverso le generazioni, visto che fra le autrici prese in considerazione ve ne sono finanche di terza. Allo stesso modo, la nazionalità o la completa padronanza linguistica talvolta possono non essere sufficienti a garantire un'identità monolitica o un'appartenenza culturale completa. Le tre scrittrici analizzate nel libro (Helen Barolini, Loise DeSalvo e Mary Cappello), pur appartenenti a tre generazioni successive, vivono tutte una diversità molteplice che finisce per diventare la cifra etica della loro scrittura: l'essere donna e il provenire da una famiglia italiana comportano lo stesso scarto rispetto alla cultura dominante americana e la possibilità di un riposizionamento critico rispetto ad essa. In maniera identica è visto il rapporto con la cultura di origine, quella patriarcale italiana, che allo stesso tempo rappresenta «il luogo della violenza e la fonte della poesia». Il rifiuto è per un'accettazione acritica della tradizione, come per una rottura radicale con le proprie origini; a livello intellettuale vi è quindi una ridefinizione continua sia rispetto alla propria comunità di appartenenza, di cui volenti o nolenti tali scrittrici sono considerate portavoce, sia rispetto alla società multiculturale americana in cui vivono, che tende ad appiattire le differenze nel talvolta banalizzante melting pot.

19 C. Romeo, Narrative tra due sponde. Memoir di italiane d'America, Roma, Carocci, 2005. 
L'affinità fra quella che comunemente era definita «letteratura dell'emigrazione» e la letteratura dell'immigrazione e postcoloniale è evidente anche analizzando il percorso dei singoli studiosi. Proprio Caterina Romeo, insieme a Cristina Lombardi-Diop - anch'essa proveniente dal contesto statunitense e autrice di un'interessante postfazione ${ }^{20}$ al romanzo Regina di fiori e di perle di Gabriella Ghermandi che associa la letteratura postcoloniale alla produzione coloniale e all'opera di Flaiano- sta attualmente dando alle stampe una miscellanea dal titolo Post-colonial Italy: the Colonial Past in Contemporary Culture ${ }^{21}$. La particolare formazione della studiosa (addottoratasi in Storia delle scritture femminili alla Sapienza e in seguito insegnante in Letterature di genere alla Rutgers University) mostra che il solco tracciato da Graziella Parati - gli studi culturali, gli studi di genere, la teoria postcoloniale, la rilettura attraverso Spivak di Gramsci e del concetto di «egemonia» - rappresenta ancora oggi un filone fervido e ricco di spunti critici.

Con le ultime opere entriamo in un territorio, per il contesto italiano, relativamente innovativo e sicuramente pieno di suggestioni soprattutto per gli spiragli che può aprire: la teoria postcoloniale non è più analizzata solo a partire dai suoi teorici «classici»o in relazione alle migrazioni e all'attuale condizione postcoloniale. Essa diventa un mezzo per rivedere anche testi più antichi, come accade a Flaiano e a tutta quella produzione che Giovanna Tomasello $^{22}$ e Maria Pagliara ${ }^{23}$ hanno definito «letteratura coloniale», e come, in forme forse più suggestive, può accadere per le opere canonizzate della letteratura italiana che oggi possono essere studiate da un'ottica differente.

L'interazione con i cultural studies porta ugualmente spunti nuovi. L'analisi dei testi filmici, ad esempio, oppure dei manifesti pubblicitari ${ }^{24}$, mostra elementi di continuità con quanto accade nella letteratura coloniale e di «propaganda», segnando un percorso preciso sui concetti di razza, identità e «italianità» che si è perpetuato fino ai giorni nostri.

20 C. Lombardi-Diop, «Postfazione», in G. Ghermandi, Regina di fiori e di perle, Roma, Donzelli, 2007.

21 C. Romeo e C. Lombardi-Diop, Postcolonial Italy : the Colonial Past in the Contemporary Culture, New York, Palgrave, 2012.

22 G. Tomasello, L'Africa tra mito e realtà, Palermo, Sellerio, 2004.

23 M. Pagliara, Il romanzo coloniale tra imperialismo e rimorso, Roma-Bari, Laterza, 2011.

24 Cfr. R. Ben-Ghiat, «Storie postcoloniali», in Narrativa, n. 33, 2012 (in corso di stampa). 
A tale proposito, agli studi di genere di Sandra Ponzanesi ${ }^{25}$, possiamo aggiungere, nel contesto anglosassone, gli interessanti lavori di Jack Andall e David Forgacs ${ }^{26}$, che seguendo la tradizione dei cultural studies di Stuart Hall riprendono alcuni aspetti specifici della postcolonialità italiana.

Anche il contesto francese ha prodotto negli ultimi anni contributi essenziali all'analisi del postcolonialismo, inteso anche qui come "condizione» postcoloniale, legato all'esperienza migratoria e all'attuale produzione contemporanea.

Alcune miscellanee tratte da convegni ospitati dalle università francesi hanno analizzato aspetti nuovi della letteratura migrante e posto l'attenzione su un approccio particolarmente interessante, perché legato, come era prevedibile, alle esperienze della francofonia. Anche in tale contesto si nota un ampliamento del corpus, segno evidente di come l'esperienza italiana, pur con le sue peculiarità, vada sempre considerata in un contesto globale di ridefinizione delle letterature nazionali e di ripensamento dell'asse ormai non più immobile che tiene uniti i concetti di lingua, nazione e identità. Nella miscellanea L'italiano lingua di migrazione ${ }^{27}$, ad esempio, emerge il concetto di «eccentricità», concepito come una scrittura combattiva che, dai margini e dalle periferie, "mina» il centro letterario. Gli scrittori eccentrici non sono semplicemente gli scrittori migranti e in un certo senso tale nozione rimette in discussione la definizione, a volte approssimativa, di scritture della migrazione o dell'emigrazione. La scrittura liminare consente, da una posizione marginale (che si manifesta attraverso l'esperienza migratoria, ma anche attraverso il genere, l'impiego del dialetto o di una lingua considerata «minore»), di ridiscutere e ridefinire la nozione di centro, cercando, proprio come accade a livello teorico in Homi Bhabha, di invertire il rapporto a prima vista univoco fra i centri e le periferie. A partire da tale concetto è possibile ripensare a tutta una serie di esperienze in seno alla letteratura italiana che non appartengono alla sfera migrante: la storia del meridione e della Sardegna, il rapporto fra i generi letterari considerati popolari e l'alta letteratura, le figure degli stranieri presenti nei romanzi degli scrittori italiani contemporanei; in tal senso le esperienze delle letterature in lingua italiana

25 S. Ponzanesi, Paradoxes of Postcolonial Cultures. Contemporary Women Writing of the Indian and the Afro-Italian Diaspora, Albany, Suny Press, 2004.

26 Cfr. Italian Colonialism: Legacy and Memory, ed. by Jack Andall e Derek Duncan, Oxford, Peter Lang, 2005.

27 Cfr. L'italiano lingua di migrazione: verso l'affermazione di una cultura transnazionale agli inizi del XXI secolo, a c. di A. Frabetti e W. Zidaric, Nantes, Les Editions du Crini, 2006. 
di emigrazione e immigrazione rappresentano un punto di vista privilegiato per approfondire la questione, ma non certo l'unico.

Un recente volume a cura di Lucia Quaquarelli apparso nel 2010, dal titolo volutamente ambiguo Certi confini ${ }^{28}$, cerca di fare il punto innanzitutto sulla definizione di queste scritture migranti, in secondo luogo sulle strategie narrative, ormai diverse e particolarmente complesse, messe in atto dagli autori per manifestare la propria alterità, e in ultimo sui legami stretti, ma alle volte conflittuali, fra letteratura migrante e letteratura postcoloniale, evidenziando all'interno di questo percorso una certa miopia della critica che, sotto lo sguardo a volte confuso della nozione di «migrazione», tendeva a cancellare o a mal riconoscere la peculiarità postcoloniale di alcune esperienze narrative. Quaquarelli si sofferma sulla necessità che, nel contesto italiano, anche gli autori non direttamente provenienti dagli antichi possedimenti coloniali partecipino a pieno diritto alla narrativa postcoloniale, poiché essi rappresentano, con le loro storie e con le loro scritture, una riflessione decisiva sull'attuale trasformazione del mondo occidentale, da intendersi proprio come risultato delle dinamiche successive alla fine della colonizzazione. Inoltre, la presenza degli autori migranti e postcoloniali nel contesto italiano ha riportato l'attenzione della critica sul concetto di «autore» e di «impegno» nella letteratura, impegno che, pur non più coincidente con l'ideologia marxista, tende comunque a considerare l'atto letterario come politico, utile in questo caso a riproporre questioni fondamentali come la memoria, la testimonianza e, in chiave si potrebbe dire quasi spivakiana, la possibilità per $\mathrm{i}$ «subalterni» di prendere la parola.

$\mathrm{Nel}$ già citato volume miscellaneo curato da Lucia Quaquarelli vi sono due articoli di Fulvio Pezzarossa e Ugo Fracassa, che rappresentano, insieme a Franca Sinopoli, fra i critici più acuti all'interno dei confini italiani. La curatela da parte di Fulvio Pezzarossa del recente volume Vent'anni di letteratura della migrazione ${ }^{29}$, atti di un convegno bolognese da lui stesso organizzato nell'ottobre del 2010, mostra la sua ricchezza nella diversità degli approcci: si va dal rapporto con il cinema e le arti visive a quello con i generi letterari, piuttosto che all'analisi del contesto editoriale ed economico in cui le opere vedono la luce. Ugo Fracassa ha, da parte sua, pubblicato un

28 Cfr. Certi confini. Sulla letteratura italiana dell'immigrazione, a c. di L. Quaquarelli, Milano, Morellini, 2010.

29 Leggere il testo e il mondo. Vent'anni di scritture della migrazione in italiano, a c. di F. Pezzarossa, Bologna, Clueb, 2012. 
volume per l'editore romano Perrone, dal titolo significativo Patria e lettere ${ }^{30}$. Che sia un italianista in Italia a soffermarsi su migrazione e postcolonialità rappresenta un tentativo di apertura «dall'interno» dell'italianistica, che cerca con fatica, all'ora attuale, di confrontarsi con la letteratura contemporanea sfuggendo i parametri e il canone consolidati. Patria $e$ lettere scinde e problematizza i termini del binomio delle Patrie lettere di Cases (già al tempo non inscindibile e oggi impossibile da ricostituire), e lo fa partendo dalla narrazione della colonia, attraverso due documentati saggi su Malaparte e su Flaiano. In particolare Flaiano si costituisce come il paradigma imprescindibile per critici ed autori migranti (Regina di fiori e di perle di Ghermandi può proporsi come una rivisitazione postcoloniale di Tempo di uccidere ${ }^{31}$ ); molto interessante risulta l'analisi lessicale di Fracassa, a partire dall'aggettivo "guasto», la cui occorrenza costante sembra quasi sfiorare l'ossessione e certo svela un «disagio» che, attraverso la lente delle teorie postcoloniali, apre una serie di riflessioni politiche sull'impresa italiana. Va forse notato, sempre a proposito di «inconscio» e di «rimosso» coloniale, come diversi autori, affrontando la colonia, abbiano fatto ricorso ad aggettivi quali «guasto», «sporco», «malandato», creando una gamma cromatico/etica dalle diverse ripercussioni socio-letterarie (e penso in particolare al Bacchelli di Mal d'Africa ${ }^{32}$, ma anche al Berto di Guerra in camicia nera ${ }^{33}$ ). La seconda parte del libro è dedicata in maniera specifica alla letteratura migrante e postcoloniale, con un saggio finale sullo stato della critica sull'argomento.

Come si è visto da questa breve analisi retrospettiva, sono ormai diversi gli approcci e le teorie che contraddistinguono la critica migrante e postcoloniale legata al contesto italiano. È una critica in più lingue, che parte da discipline diverse (dagli studi di genere alla letteratura comparata fino all'italianistica) e che è prodotta in paesi diversi (oltre ai già citati Francia, Italia, Inghilterra e Stati Uniti possono aggiungersi studiosi in Turchia, Australia, Germania, Brasile e Canada). Al di là della difficoltà che hanno avuto questi studi a prendere piede nel contesto accademico, è fuori di dubbio che si sia oggi incrinato l'argine che teneva unite lingua e letteratura nazionale e che l'approccio alla letteratura italiana, a partire dalle teorizzazioni citate, mostra alcuni aspetti innovativi.

30 U. Fracassa, Patria e lettere, Roma, Perrone, 2012.

31 E. Flaiano, Tempo di uccidere, Milano, Longanesi, 1947 (1a ed.), Milano, Rizzoli, 2000.

32 R. Bacchelli, Mal d'Africa, Milano, Treves, Treccani, 1934 (1a ed.), Milano, Rizzoli, 1990.

33 G. Berto, Guerra in camicia nera, (Coll. Vita vissuta), Milano, Garzanti, 1967. 